\title{
A Homological Invariant of Certain Torsion Free Crystallographic Groups
}

\author{
Hazzirah Izzati Mat Hassim ${ }^{1^{*}}$, Nor Haniza Sarmin ${ }^{1}$ and Nor Muhainiah Mohd $\operatorname{Ali}^{1}$ \\ ${ }^{1}$ Department of Mathematical Sciences, Faculty of Science, Universiti Teknologi Malaysia, 81310 \\ UTM Johor Bahru, Johor, Malaysia
}

\begin{abstract}
Several homological invariants namely the nonabelian tensor square, the exterior square and the Schur multiplier of groups have been of research interests by group theorists over the years. Besides, there are also some other homological invariants which can be deduced from these invariants, as example, the central subgroup of the nonabelian tensor square of a group $G$, known as $\nabla(G)$. The computations of the homological invariants of crystallographic groups strengthen the link between group theory with crystallography theory. In this paper, $\nabla(G)$ is determined for certain torsion free crystallographic groups focusing on those with cyclic point groups of order three and five.
\end{abstract}

Keywords: homological invariant, nonabelian tensor square, crystallographic

\section{INTRODUCTION}

A central subgroup of the nonabelian tensor square of a group $G$, namely $\nabla(G)$ is one of the important structures in the development of other homological invariants of that group including the exterior square and Schur multiplier. In some cases, the nonabelian tensor square itself can be determined by using $\nabla(G)$. Researches have been conducted on the computation of $\nabla(G)$ for various groups $G$ including in (Abdul Ladi et al., 2017), (Mohd Idrus et al., 2015) and (Mohammad et al., 2016). A Bieberbach group is an extension of a free abelian group known as lattice subgroup with a finite point group.In this paper, $\nabla(G)$ is computed for certain torsion free crystallographic groups known as Bieberbach groups. Focus is given to the Bieberbach group with cyclic point groups of order three and five.

\section{METHODS}

Ellis and Leonard in (Ellis\& Leonard, 1995) discovered the relation between the nonabelian tensor square of a group $G$ with the commutator $\left[G, G^{\varphi}\right]$ as given in the following theorem.

Theorem 1.(Ellis \& Leonard, 1995) Let $G$ be a group. The map $\sigma: G \otimes G \rightarrow\left[G, G^{\varphi}\right] \triangleleft \nu(G)$ defined by $\sigma(g \otimes h)=\left[g, h^{\varphi}\right]$ for all $g, h$ in $G$ is an isomorphism.

By using the preceding theorem as a basis and the matrix presentation obtained from Crystallographic, Algorithms and Tables (CARAT) package (CARAT homepage), the polycyclic presentations of all Bieberbach groups with cyclic point group of order three, $C_{3}$, (up to dimension six) are constructed in (Mat Hassim, 2014).The polycyclic presentations of all nonisomorphic Bieberbach groups with point group $C_{3}$ are listed as follows:

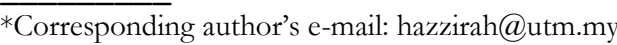




$$
\begin{aligned}
& H_{1}(3)=\left\langle a, l_{1}, l_{2}, l_{3}\right| a^{3}=l_{3},{ }^{a} l_{1}=l_{1}^{-1} l_{2}^{-1},{ }^{a} l_{2}=l_{1} \text {, } \\
& \left.{ }^{a} l_{3}=l_{3},{ }^{l_{1}} l_{2}=l_{2},{ }^{1} l_{3}=l_{3},{ }^{l_{2}} l_{3}=l_{3}\right\rangle, \\
& H_{2}(4)=\left\langle a, l_{1}, l_{2}, l_{3}, l_{4}\right| a^{3}=l_{3},{ }^{a} l_{1}=l_{2}^{-1} \text {, } \\
& { }^{a} l_{2}=l_{1} l_{2}^{-1},{ }^{a} l_{3}=l_{3},{ }^{a} l_{4}=l_{4} \text {, } \\
& { }^{l_{1}} l_{2}=l_{2},{ }^{l_{1}} l_{3}=l_{3},{ }^{1} l_{4}=l_{4} \text {, } \\
& \left.{ }^{l_{2}} l_{3}=l_{3},{ }^{l_{2}} l_{4}=l_{4},{ }^{l_{3}} l_{4}=l_{4}\right\rangle \text {, } \\
& H_{3}(4)=\left\langle a, l_{1}, l_{2}, l_{3}, l_{4}\right| a^{3}=l_{1},{ }^{a} l_{1}=l_{1} \text {, } \\
& { }^{a} l_{2}=l_{4}^{-1},{ }^{a} l_{3}=l_{2},{ }^{a} l_{4}=l_{3}^{-1}, \\
& { }^{l_{1}} l_{2}=l_{2},{ }^{1} l_{3}=l_{3},{ }^{1} l_{4}=l_{4} \text {, } \\
& \left.{ }^{l_{2}} l_{3}=l_{3},{ }^{l_{2}} l_{4}=l_{4},{ }^{l_{3}} l_{4}=l_{4}\right\rangle \text {, } \\
& H_{4}(5)=\left\langle a, l_{1}, l_{2}, l_{3}, l_{4}, l_{5}\right| a^{3}=l_{5},{ }^{a} l_{1}=l_{1}^{-1} l_{2}^{-1} \text {, } \\
& { }^{a} l_{2}=l_{1},{ }^{a} l_{3}=l_{3}^{-1} l_{4}^{-1},{ }^{a} l_{4}=l_{3},{ }^{a} l_{5}=l_{5}, \\
& { }^{l_{1}} l_{2}=l_{2},{ }^{l_{1}} l_{3}=l_{3},{ }^{l_{1}} l_{4}=l_{4},{ }_{1} l_{5}=l_{5} \text {, } \\
& { }^{l_{2}} l_{3}=l_{3},{ }^{l_{2}} l_{4}=l_{4},{ }^{l_{2}} l_{5}=l_{5},{ }^{l_{3}} l_{4}=l_{4}, \\
& \left.{ }^{l_{3}} l_{5}=l_{5},{ }^{l_{4}} l_{5}=l_{5}\right\rangle \text {, }
\end{aligned}
$$

$$
\begin{gathered}
H_{5}(5)=\left\langle a, l_{1}, l_{2}, l_{3}, l_{4}, l_{5}\right| a^{3}=l_{3},{ }^{a} l_{1}=l_{1}^{-1} l_{2}^{-1}, \\
{ }^{a} l_{2}=l_{1},{ }^{a} l_{3}=l_{3},{ }^{a} l_{4}=l_{4},{ }^{a} l_{5}=l_{5}, \\
{ }^{l_{1}} l_{2}=l_{2},{ }^{l_{1}} l_{3}=l_{3},{ }^{l_{1}} l_{4}=l_{4},{ }^{l_{1}} l_{5}=l_{5}, \\
{ }^{l_{2}} l_{3}=l_{3},{ }^{l_{2}} l_{4}=l_{4},{ }^{l_{2}} l_{5}=l_{5},{ }^{l_{3}} l_{4}=l_{4}, \\
\left.{ }^{{ }^{3}} l_{5}=l_{5},{ }^{l_{4}} l_{5}=l_{5}\right\rangle,
\end{gathered}
$$$$
H_{6}(5)=\left\langle a, l_{1}, l_{2}, l_{3}, l_{4}, l_{5}\right| a^{3}=l_{2},{ }^{a} l_{1}=l_{1},{ }^{a} l_{2}=l_{2} \text {, }
$$$$
{ }^{a} l_{3}=l_{5}^{-1},{ }^{a} l_{4}=l_{3}^{-1},{ }^{a} l_{5}=l_{4},{ }^{1} l_{2}=l_{2},
$$$$
{ }^{l_{1}} l_{3}=l_{3},{ }^{1} l_{4}=l_{4},{ }^{1} l_{5}=l_{5},{ }^{l_{2}} l_{3}=l_{3} \text {, }
$$$$
{ }^{l_{2}} l_{4}=l_{4},{ }^{l_{2}} l_{5}=l_{5},{ }^{l_{3}} l_{4}=l_{4},{ }^{l^{3}} l_{5}=l_{5} \text {, }
$$$$
\left.{ }^{l_{4}} l_{5}=l_{5}\right\rangle \text {, }
$$$$
H_{7}(6)=\left\langle a, l_{1}, l_{2}, l_{3}, l_{4}, l_{5}, l_{6}\right| a^{3}=l_{5},{ }^{a} l_{1}=l_{1}^{-1} l_{2}^{-1} \text {, }
$$$$
{ }^{a} l_{2}=l_{1},{ }^{a} l_{3}=l_{3}^{-1} l_{4},{ }^{a} l_{4}=l_{3}^{-1},{ }^{a} l_{5}=l_{5},
$$$$
{ }^{a} l_{6}=l_{6},{ }^{l_{1}} l_{2}=l_{2},{ }^{l_{1}} l_{3}=l_{3},{ }^{{ }_{1}} l_{4}=l_{4},
$$$$
{ }^{l_{1}} l_{5}=l_{5},{ }^{l_{1}} l_{6}=l_{6},{ }^{l_{2}} l_{3}=l_{3},{ }^{l_{2}} l_{4}=l_{4} \text {, }
$$$$
{ }^{l_{2}} l_{5}=l_{5},{ }^{l_{2}} l_{6}=l_{6},{ }^{3} l_{4}=l_{4},{ }^{l_{3}} l_{5}=l_{5} \text {, }
$$$$
\left.{ }^{l_{3}} l_{6}=l_{6},{ }^{l_{4}} l_{5}=l_{5},{ }^{l_{4}} l_{6}=l_{6},{ }^{l_{5}} l_{6}=l_{6}\right\rangle \text {, }
$$

$$
\begin{aligned}
& H_{8}(6)=\left\langle a, l_{1}, l_{2}, l_{3}, l_{4}, l_{5}, l_{6}\right| a^{3}=l_{1},{ }^{a} l_{1}=l_{1} \text {, } \\
& { }^{a} l_{2}=l_{2}{ }^{1} l_{3},{ }^{a} l_{3}=l_{2}{ }^{1},{ }^{a} l_{4}=l_{5}{ }^{1}, \\
& { }^{a} l_{5}=l_{6}{ }^{1},{ }^{a} l_{6}=l_{4},{ }^{l_{1}} l_{2}=l_{2},{ }^{l_{1}} l_{3}=l_{3}, \\
& { }^{l_{1}} l_{4}=l_{4},{ }^{{ }^{1}} l_{5}=l_{5},{ }^{l_{1}} l_{6}=l_{6},{ }^{l_{2}} l_{3}=l_{3}, \\
& { }^{l_{2}} l_{4}=l_{4},{ }^{l^{2}} l_{5}=l_{5},{ }^{l_{2}} l_{6}=l_{6},{ }^{l^{3}} l_{4}=l_{4}, \\
& { }^{l_{3}} l_{5}=l_{5},{ }^{l_{3}} l_{6}=l_{6},{ }^{l^{4}} l_{5}=l_{5},{ }^{l^{4}} l_{6}=l_{6} \text {, } \\
& \left.{ }^{l_{5}} l_{6}=l_{6}\right\rangle \text {, } \\
& H_{9}(6)=\left\langle a, l_{1}, l_{2}, l_{3}, l_{4}, l_{5}, l_{6}\right| a^{3}=l_{3},{ }^{a} l_{1}=l_{1}{ }^{1} l_{2}{ }^{1} \text {, } \\
& { }^{a} l_{2}=l_{1},{ }^{a} l_{3}=l_{3},{ }^{a} l_{4}=l_{4},{ }^{a} l_{5}=l_{5}, \\
& { }^{a} l_{6}=l_{6},{ }^{l_{1}} l_{2}=l_{2},{ }^{l_{1}} l_{3}=l_{3},{ }^{l_{1}} l_{4}=l_{4}, \\
& { }^{l_{1}} l_{5}=l_{5},{ }_{1} l_{6}=l_{6},{ }^{l_{2}} l_{3}=l_{3},{ }^{l_{2}} l_{4}=l_{4} \text {, } \\
& { }^{l_{2}} l_{5}=l_{5},{ }^{l_{2}} l_{6}=l_{6},{ }^{l_{3}} l_{4}=l_{4},{ }^{l^{3}} l_{5}=l_{5} \text {, } \\
& \left.{ }^{l_{3}} l_{6}=l_{6},{ }^{l}{ }^{2} l_{5}=l_{5},{ }^{l^{4}} l_{6}=l_{6},{ }^{{ }^{5}} l_{6}=l_{6}\right\rangle \text {, } \\
& H_{10}(6)=\left\langle a, l_{1}, l_{2}, l_{3}, l_{4}, l_{5}, l_{6}\right| a^{3}=l_{2},{ }^{a} l_{1}=l_{1} \text {, } \\
& { }^{a} l_{2}=l_{2},{ }^{a} l_{3}=l_{3},{ }^{a} l_{4}=l_{6}{ }^{1},{ }^{a} l_{5}=l_{4}{ }^{1}, \\
& { }^{a} l_{6}=l_{5},{ }_{1} l_{2}=l_{2},{ }^{l_{1}} l_{3}=l_{3},{ }^{l_{1}} l_{4}=l_{4} \text {, } \\
& { }^{l_{1}} l_{5}=l_{5},{ }_{1} l_{6}=l_{6},{ }^{l_{2}} l_{3}=l_{3},{ }^{l_{2}} l_{4}=l_{4} \text {, } \\
& { }^{l_{2}} l_{5}=l_{5},{ }^{l_{2}} l_{6}=l_{6},{ }^{l_{3}} l_{4}=l_{4},{ }^{l_{3}} l_{5}=l_{5} \text {, } \\
& \left.{ }^{l^{3}} l_{6}=l_{6},{ }^{l_{4}} l_{5}=l_{5},{ }^{l^{4}} l_{6}=l_{6},{ }^{l_{5}} l_{6}=l_{6}\right\rangle \text {. }
\end{aligned}
$$

Based on (Mat Hassim, 2014), the Bieberbach groups $H_{5}(5)$ and $H_{9}(6)$ belong to the same family as $H_{1}(3)$. Hence, the properties of these two groups coincide with the properties of $H_{1}(3)$. Next, the derived subgroups and the abelianisations of the Bieberbach groups with point group $C_{3}$ are presented in the next two propositions.

Proposition 1. (Mat Hassim et al., 2014) The derived subgroups of the Bieberbach groups with point group $C_{3}$ are given as follows : 


$$
\begin{aligned}
& H_{1}(3)^{\prime}=\left\langle l_{1} l_{2}^{-1}, l_{1}^{-2} l_{2}^{-1}\right\rangle \cong C_{0}^{2}, \\
& H_{2}(4)^{\prime}=\left\langle l_{1}^{-1} l_{2}^{-1}, l_{1} l_{2}^{-2}\right\rangle \cong C_{0}^{2}, \\
& H_{3}(4)^{\prime}=\left\langle l_{2} l_{3}^{-1}, l_{2}^{-1} l_{4}^{-1}\right\rangle \cong C_{0}^{2}, \\
& H_{4}(5)^{\prime}=\left\langle l_{1} l_{2}^{-1}, l_{1}^{-2} l_{2}^{-1}, l_{3} l_{4}^{-1}, l_{3}^{-2} l_{4}^{-1}\right\rangle \cong C_{0}^{4}, \\
& H_{6}(5)^{\prime}=\left\langle l_{3}^{-1} l_{4}^{-1}, l_{4} l_{5}^{-1}\right\rangle \cong C_{0}^{2}, \\
& H_{7}(6)^{\prime}=\left\langle l_{1} l_{2}^{-1}, l_{1}^{-2} l_{2}^{-1}, l_{3}^{-1} l_{4}^{-1}, l_{3}^{-2} l_{4}\right\rangle \cong C_{0}^{4}, \\
& H_{8}(6)^{\prime}=\left\langle l_{2}^{-1} l_{3}^{-1}, l_{2}^{-2} l_{3}, l_{4}^{-1} l_{5}^{-1}, l_{4} l_{6}^{-1}\right\rangle \cong C_{0}^{4}, \\
& H_{10}(6)^{\prime}=\left\langle l_{4}^{-1} l_{5}^{-1}, l_{5} l_{6}^{-1}\right\rangle \cong C_{0}^{2} .
\end{aligned}
$$

Proposition 2. (Mat Hassim et al., 2014)The abelianisation of the Bieberbach groups with point group $C_{3}$ are as in the following :

$$
\begin{aligned}
H_{1}(3)^{a b} & =\left\langle a H_{1}(3)^{\prime}, l_{1} H_{1}(3)^{\prime}\right\rangle \cong C_{0} \times C_{3}, \\
H_{2}(4)^{a b} & =\left\langle a H_{2}(4)^{\prime}, l_{1} H_{2}(4)^{\prime}, l_{4} H_{2}(4)^{\prime}\right\rangle \cong C_{0} \times C_{3} \times C_{0}, \\
H_{3}(4)^{a b} & =\left\langle a H_{3}(4)^{\prime}, l_{2} H_{3}(4)^{\prime}\right\rangle \cong C_{0}^{2}, \\
H_{4}(5)^{a b} & =\left\langle a H_{4}(5)^{\prime}, l_{1} H_{4}(5)^{\prime}, l_{3} H_{4}(5)^{\prime}\right\rangle \cong C_{0} \times C_{3} \times C_{3}, \\
H_{6}(5)^{a b} & =\left\langle a H_{6}(5)^{\prime}, l_{1} H_{6}(5)^{\prime}, l_{3} H_{6}(5)^{\prime}\right\rangle \cong C_{0}^{3}, \\
H_{7}(6)^{a b} & =\left\langle a H_{7}(6)^{\prime}, l_{1} H_{7}(6)^{\prime}, l_{3} H_{7}(6)^{\prime}, l_{6} H_{7}(6)^{\prime}\right\rangle \\
& \cong C_{0} \times C_{3} \times C_{3} \times C_{0}, \\
H_{8}(6)^{a b} & =\left\langle a H_{8}(6)^{\prime}, l_{2} H_{8}(6)^{\prime}, l_{4} H_{8}(6)^{\prime}\right\rangle \cong C_{0} \times C_{3} \times C_{0}, \\
H_{10}(6)^{a b} & =\left\langle a H_{10}(6)^{\prime}, l_{1} H_{10}(6)^{\prime}, l_{3} H_{10}(6)^{\prime}, l_{4} H_{10}(6)^{\prime}\right\rangle \\
& \cong C_{0}^{4} .
\end{aligned}
$$

In addition, the preliminary results from previous researches which are used in the computation of $\nabla(G)$ of the chosen Bieberbach groups are also included in this section.

Proposition 3. (Blyth et al., 2010) Let $G$ be a group such that $G^{a b}$ is finitely generated. Assume that $G^{a b}$ is the direct product of the cyclic groups $\left\langle x_{i} G^{\prime}\right\rangle$, for $i=1, \ldots, s$ and set $E(G)$ to be $\left\langle\left[x_{i}, x_{j}^{\varphi}\right] \mid i<j\right\rangle\left[G, G^{\prime \varphi}\right]$. Then the following hold:

1. $\nabla(G)$ is generated by the elements of the set

$$
\left\{\left[x_{i}, x_{i}^{\varphi}\right],\left[x_{i}, x_{j}^{\varphi}\right]\left[x_{j}, x_{i}^{\varphi}\right] \mid 1 \leq i<j \leq s\right\} ;
$$

2. $\left[G, G^{\varphi}\right]=\nabla(G) E(G)$.

Proposition 4. (Blyth et al., 2010; Blyth \& Morse, 2009)Let $g$ and $h$ be elements of $G$ such that $[g, h]=1$. Then in $v(G)$,

1. $\left[g^{n}, h^{\varphi}\right]=\left[g, h^{\varphi}\right]^{n}=\left[g,\left(h^{\varphi}\right)^{n}\right]$ for all integers $n$;

2. $\left[g^{n},\left(h^{m}\right)\right]\left[h^{m},\left(g^{n}\right)\right]=([g, h][h, g])^{n m}$;

3. $[g, h]$ is in the center of $(G)$

Proposition 5. (Masri, 2009) Let $G$ be a group and $a, b, c \in G$. Then, $\left[[a, b], c^{\varphi}\right]=\left[c,[a, b]^{\varphi}\right]^{-1}$ in $v(G)$.

Proposition 6. (Masri, 2009) Let $G$ and $H$ be groups and let $g \in G$. Suppose $\phi$ is a homomorphism from $G$ onto H.If $\phi(g)$ has finite order then $|\phi(g)|$ divides $|g|$. Otherwise the order of $\phi(g)$ equals the order of $g$.

Proposition 7. (Zomorodian, 2005) Let $A, B$ and $C$ be abelian groups and $C_{0}$ is the infinite cyclic group. Consider the ordinary tensor product of two abelian groups. Then,

1. $C_{0} \otimes A \cong A$,

2. $C_{0} \otimes C_{0} \cong C_{0}$,

3. $C_{n} \otimes C_{m} \cong C_{g c d(n, m)}$, for $n, m \in \mathrm{Z}$, and

4. $A \otimes(B \times C)=(A \otimes B) \times(A \otimes C)$.

Theorem 2. (Brown et al., 1987) Let $G$ and $H$ be groups such that there is an epimorphism $\epsilon: G \rightarrow H$. Then there exists an epimorphism

$$
\alpha: G \otimes G \rightarrow H \otimes H
$$

defined by $\alpha(g \otimes h)=\epsilon(g) \otimes \epsilon(h)$.

\section{RESULTS AND DISCUSSIONS}

This section is divided into two parts; the first part is on the computation of $\nabla(G)$ of the Bieberbach groups with point group $C_{3}$, namely $H_{i}(j)$, while the second part consists the computation of $\nabla(G)$ of the Bieberbach 
groups with point group $C_{5}$, denoted as $N_{i}(j)$.

\section{A. The Homological Invariant $\nabla(G)$ of the}

Bieberbach Groups with Point Group $C_{3}$

The computation of $\nabla\left(H_{1}(3)\right)$ is presented in the following theorem.

Theorem 3. Let $H_{1}(3)$ be a Bieberbach group with point group $C_{3}$ of dimension three. Then,

$$
\begin{aligned}
\nabla\left(H_{1}(3)\right) & =\left\langle a \otimes a, l_{1} \otimes l_{1},\left(a \otimes l_{1}\right)\left(l_{1} \otimes a\right)\right\rangle \\
& \cong C_{0} \times C_{3} \times C_{3} .
\end{aligned}
$$

\section{Proof.}

By Proposition 2, the abelianisation of $H_{1}(3)$ is generated by $a H_{1}(3)^{\prime}$ and $l_{1} H_{1}(3)^{\prime}$ of infinite order and order three, respectively. Then, based on Proposition 3 (i), $\nabla\left(H_{1}(3)\right)$ is generated by $\left[a, a^{\varphi}\right],\left[l_{1}, l_{1}^{\varphi}\right]$ and $\left[a, l_{1}^{\varphi}\right]\left[l_{1}, a^{\varphi}\right]$. Since $\nabla\left(H_{1}(3)\right)$ is a subgroup of $H_{1}(3) \otimes H_{1}(3)$ and the mapping $\sigma: H_{1}(3) \otimes H_{1}(3) \rightarrow\left[H_{1}(3), H_{1}(3)^{\varphi}\right] \quad$ defined by $\sigma(g \otimes h)=\left[g, h^{\varphi}\right] \quad$ for $\quad$ all $\quad g, h \in H_{1}(3) \quad$ is anisomorphism, thus

$$
\nabla\left(H_{1}(3)\right)=\left\langle a \otimes a, l_{1} \otimes l_{1},\left(a \otimes l_{1}\right)\left(l_{1} \otimes a\right)\right\rangle .
$$

Next, the order of each of the generators of $\nabla\left(H_{1}(3)\right)$ is computed. By Proposition $1, l_{1}^{3}$ is in $H_{1}(3)^{\prime}$. Hence,by the identities of commutator, $\left[l_{1}, l_{1}^{\varphi}\right]^{9}=\left[l_{1}^{3},\left(l_{1}^{3}\right)^{\varphi}\right]=1$. Therefore, $\left[l_{1}, l_{1}^{\varphi}\right]$ has order dividing 9. Suppose the order of $\left[l_{1}, l_{1}^{\varphi}\right]$ is 9. Then, there is no positive integer $r$ smaller than 9, such that $\left[l_{1}, l_{1}^{\varphi}\right]^{r}=1$. However, since $l_{1}^{3} \in H_{1}(3)^{\prime}$, then by Proposition 5, $\left[l_{1},\left(l_{1}^{3}\right)^{\varphi}\right]=\left[l_{1}^{3}, l_{1}^{\varphi}\right]^{-1}$. This implies that $\left[l_{1},\left(l_{1}^{3}\right)^{\varphi}\right]\left[l_{1}^{3}, l_{1}^{\varphi}\right]=1 . \quad$ By $\quad$ Proposition 4(i), $\left[l_{1}, l_{1}^{\varphi}\right]^{3}\left[l_{1}, l_{1}^{\varphi}\right]^{3}=1$. Hence, it is found that $\left[l_{1}, l_{1}^{\varphi}\right]^{6}=1$, a contradiction. Thus, the order of $\left[l_{1}, l_{1}^{\varphi}\right]$ cannot be 9 . Since the greatest common divisor of 6 and 9 is 3 , then, the order of $\left[l_{1}, l_{1}^{\varphi}\right]$ is 3 .

Next, the order of $\left[a, l_{1}^{\varphi}\right]\left[l_{1}, a^{\varphi}\right]$ is also 3 based on the commutator identities and Proposition 5;

$$
\begin{aligned}
\left(\left[a, l_{1}^{\varphi}\right]\left[l_{1}, a^{\varphi}\right]\right)^{3} & =\left[a,\left(l_{1}^{3}\right)^{\varphi}\right]\left[l_{1}^{3}, a^{\varphi}\right] \\
& =\left[a,\left(l_{1}^{3}\right)^{\varphi}\right]\left[a,\left(l_{1}^{3}\right)^{\varphi}\right]^{-1} \\
& =1 .
\end{aligned}
$$

The abelianisation of $H_{1}(3)$ is denoted by $H_{1}(3)^{a b}$ with natural homomorphism $\epsilon: H_{1}(3) \rightarrow H_{1}(3)^{a b}$. Since $H_{1}(3)^{a b}$ is finitely generated, then its nonabelian tensor square is simply the ordinary tensor product of $H_{1}(3)^{a b} \cong C_{0} \times C_{3}$. By Proposition 7,

$$
\begin{aligned}
H_{1}(3)^{a b} \otimes H_{1}(3)^{a b} & \cong\left(C_{0} \times C_{3}\right) \otimes\left(C_{0} \times C_{3}\right) \\
& \cong C_{0} \times C_{3} \times C_{3} \times C_{3} .
\end{aligned}
$$

Then, Proposition 2 provides that $H_{1}(3)^{a b}$ is generated by $\epsilon(a)$ and $\epsilon\left(l_{1}\right)$ of infinite order and order three, respectively. Again, by Proposition 7, $\langle\epsilon(a) \otimes \epsilon(a)\rangle \cong C_{0},\left\langle\epsilon(a) \otimes \epsilon\left(l_{1}\right)\right\rangle \cong C_{3}$, $\left\langle\epsilon\left(l_{1}\right) \otimes \epsilon(a)\right\rangle \cong C_{3}, \quad$ and $\quad\left\langle\epsilon\left(l_{1}\right) \otimes \epsilon\left(l_{1}\right)\right\rangle \cong C_{3}$. By Theorem 2, there is a natural epimorphism

$$
\alpha: H_{1}(3) \otimes H_{1}(3) \rightarrow H_{1}(3)^{a b} \otimes H_{1}(3)^{a b} .
$$

Therefore, the image $\alpha(a \otimes a)=\epsilon(a) \otimes \epsilon(a)$ has infinite order. Thus by Proposition 6, $a \otimes a$ has also infinite order. Finally, the desired result is obtained, which is

$$
\begin{aligned}
\nabla\left(H_{1}(3)\right) & =\left\langle a \otimes a, l_{1} \otimes l_{1},\left(a \otimes l_{1}\right)\left(l_{1} \otimes a\right)\right\rangle \\
& \cong C_{0} \times C_{3} \times C_{3} .
\end{aligned}
$$

Using similar method as above, $\nabla\left(H_{i}(j)\right)$ can be computed for other Bieberbach groups with point group $C_{3}$. The results are summarized in the following table: 
Table 1: The homological functor $\nabla\left(H_{i}(j)\right)$

\begin{tabular}{|c|c|c|}
\hline$H_{i}(j)$ & $\nabla\left(H_{i}(j)\right)$ & $\cong$ \\
\hline$H_{1}(3)$ & $\left\langle a \otimes a, l_{1} \otimes l_{1},\left(a \otimes l_{1}\right)\left(l_{1} \otimes a\right)\right\rangle$ & $\begin{array}{c}C_{0} \times C_{3} \\
\times C_{3}\end{array}$ \\
\hline$H_{2}(4)$ & $\begin{array}{c}\left\langle a \otimes a, l_{1} \otimes l_{1}, l_{4} \otimes l_{4}\right. \\
\left(a \otimes l_{1}\right)\left(l_{1} \otimes a\right),\left(a \otimes l_{4}\right)\left(l_{4} \otimes a\right) \\
\left.\left(l_{1} \otimes l_{4}\right)\left(l_{4} \otimes l_{1}\right)\right\rangle\end{array}$ & $C_{0}^{3} \times C_{3}^{3}$ \\
\hline$H_{3}(4)$ & $\left\langle a \otimes a, l_{2} \otimes l_{2},\left(a \otimes l_{2}\right)\left(l_{2} \otimes a\right)\right\rangle$ & $C_{0}^{3}$ \\
\hline$H_{4}(5)$ & $\begin{array}{c}\left\langle a \otimes a, l_{1} \otimes l_{1}, l_{3} \otimes l_{3}\right. \\
\left(a \otimes l_{1}\right)\left(l_{1} \otimes a\right),\left(a \otimes l_{3}\right)\left(l_{3} \otimes a\right) \\
\left.\left(l_{1} \otimes l_{3}\right)\left(l_{3} \otimes l_{1}\right)\right\rangle\end{array}$ & $C_{0} \times C_{3}^{5}$ \\
\hline$H_{6}(5)$ & $\begin{array}{c}\left\langle a \otimes a, l_{1} \otimes l_{1}, l_{3} \otimes l_{3}\right. \\
\left(a \otimes l_{1}\right)\left(l_{1} \otimes a\right),\left(a \otimes l_{3}\right)\left(l_{3} \otimes a\right) \\
\left.\left(l_{1} \otimes l_{3}\right)\left(l_{3} \otimes l_{1}\right)\right\rangle\end{array}$ & $C_{0}^{6}$ \\
\hline$H_{7}(6)$ & $\begin{array}{c}\left\langle a \otimes a, l_{1} \otimes l_{1}, l_{3} \otimes l_{3}, l_{6} \otimes l_{6}\right. \\
\left(a \otimes l_{1}\right)\left(l_{1} \otimes a\right),\left(a \otimes l_{3}\right)\left(l_{3} \otimes a\right) \\
\left(a \otimes l_{6}\right)\left(l_{6} \otimes a\right),\left(l_{1} \otimes l_{3}\right)\left(l_{3} \otimes l_{1}\right) \\
\left(l_{1} \otimes l_{6}\right)\left(l_{6} \otimes l_{1}\right) \\
\left.\left(l_{3} \otimes l_{6}\right)\left(l_{6} \otimes l_{3}\right)\right\rangle\end{array}$ & $C_{0}^{3} \times C_{3}^{7}$ \\
\hline$H_{8}(6)$ & $\begin{array}{c}\left\langle a \otimes a, l_{2} \otimes l_{2}, l_{4} \otimes l_{4}\right. \\
\left(a \otimes l_{2}\right)\left(l_{2} \otimes a\right),\left(a \otimes l_{4}\right)\left(l_{4} \otimes a\right) \\
\left.\left(l_{2} \otimes l_{4}\right)\left(l_{4} \otimes l_{2}\right)\right\rangle\end{array}$ & $C_{0}^{3} \times C_{3}^{3}$ \\
\hline$H_{10}(6)$ & $\begin{array}{c}\left\langle a \otimes a, l_{1} \otimes l_{1}, l_{3} \otimes l_{3}, l_{4} \otimes l_{4}\right. \\
\left(a \otimes l_{1}\right)\left(l_{1} \otimes a\right),\left(a \otimes l_{3}\right)\left(l_{3} \otimes a\right) \\
\left(a \otimes l_{4}\right)\left(l_{4} \otimes a\right),\left(l_{1} \otimes l_{3}\right)\left(l_{3} \otimes l_{1}\right) \\
\left(l_{1} \otimes l_{4}\right)\left(l_{4} \otimes l_{1}\right) \\
\left.\left(l_{3} \otimes l_{4}\right)\left(l_{4} \otimes l_{3}\right)\right\rangle\end{array}$ & $C_{0}^{10}$ \\
\hline
\end{tabular}

\section{B. The Homological Invariant $\nabla(G)$ of the}

Bieberbach Groups with Point Group $C_{5}$

The polycyclic presentations of all non-isomorphic Bieberbach groups with cyclic point group of order five, up to dimension six are given in this subsection. The
CARAT package shows that there are three Bieberbach groups with cyclic point group of order $5, C_{5}$, up to dimension six. Using the definition of the polycyclic presentation, the constructed polycyclic presentations of these Bieberbach groups are given as follows:

$$
\begin{gathered}
N_{1}(5)=\left\langle a, l_{1}, l_{2}, l_{3}, l_{4}, l_{5}\right| a^{5}=l_{5},{ }^{a} l_{1}=l_{4}^{-1}, \\
{ }^{a} l_{2}=l_{2}^{-1} l_{3}^{-1},{ }^{a} l_{3}=l_{1},{ }^{a} l_{4}=l_{1} l_{2}^{-1}, \\
{ }^{a} l_{5}=l_{5},{ }^{l_{1}} l_{2}=l_{2},{ }^{1} l_{3}=l_{3},{ }^{1} l_{4}=l_{4}, \\
{ }^{{ }^{1}} l_{5}=l_{5},{ }^{l_{2}} l_{3}=l_{3},{ }^{{ }^{2}} l_{4}=l_{4},{ }^{l_{2}} l_{5}=l_{5}, \\
\left.{ }^{{ }^{3}} l_{4}=l_{4},{ }^{l_{3}} l_{5}=l_{5},{ }^{l_{4}} l_{5}=l_{5}\right\rangle,
\end{gathered}
$$

$$
\begin{aligned}
& N_{2}(6)=\left\langle a, l_{1}, l_{2}, l_{3}, l_{4}, l_{5}, l_{6}\right| a^{5}=l_{5},{ }^{a} l_{1}=l_{4} \text {, } \\
& { }^{a} l_{2}=l_{1} l_{2}^{-1} l_{3}^{-1},{ }^{a} l_{3}=l_{1}^{-1} l_{2},{ }^{a} l_{4}=l_{2}^{-1}, \\
& { }^{a} l_{5}=l_{5},{ }^{a} l_{6}=l_{6},{ }^{l_{1}} l_{2}=l_{2},{ }^{l_{1}} l_{3}=l_{3} \text {, } \\
& { }^{l_{1}} l_{4}=l_{4},{ }^{l_{1}} l_{5}=l_{5},{ }_{1} l_{6}=l_{6},{ }^{l_{2}} l_{3}=l_{3} \text {, } \\
& { }^{l_{2}} l_{4}=l_{4},{ }^{l_{2}} l_{5}=l_{5},{ }^{l_{2}} l_{6}=l_{6},{ }^{l_{3}} l_{4}=l_{4}, \\
& { }^{l^{3}} l_{5}=l_{5},{ }^{l_{3}} l_{6}=l_{6},{ }^{l_{4}} l_{5}=l_{5},{ }^{l_{4}} l_{6}=l_{6} \text {, } \\
& \left.{ }^{l_{5}} l_{6}=l_{6}\right\rangle \text {, } \\
& N_{3}(6)=\left\langle a, l_{1}, l_{2}, l_{3}, l_{4}, l_{5}, l_{6}\right| a^{5}=l_{1},{ }^{a} l_{1}=l_{1} \text {, } \\
& { }^{a} l_{2}=l_{4},{ }^{a} l_{3}=l_{5}^{-1},{ }^{a} l_{4}=l_{6},{ }^{a} l_{5}=l_{2}^{-1}, \\
& { }^{a} l_{6}=l_{3},{ }^{l_{1}} l_{2}=l_{2},{ }^{l_{1}} l_{3}=l_{3},{ }^{l_{1}} l_{4}=l_{4}, \\
& { }^{l_{1}} l_{5}=l_{5},{ }^{l_{1}} l_{6}=l_{6},{ }^{l_{2}} l_{3}=l_{3},{ }^{l_{2}} l_{4}=l_{4} \text {, } \\
& { }^{l_{2}} l_{5}=l_{5},{ }^{l_{2}} l_{6}=l_{6},{ }^{l_{3}} l_{4}=l_{4},{ }^{l_{3}} l_{5}=l_{5} \text {, } \\
& \left.{ }^{l^{3}} l_{6}=l_{6},{ }^{l_{4}} l_{5}=l_{5},{ }^{l_{4}} l_{6}=l_{6},{ }^{l_{5}} l_{6}=l_{6}\right\rangle \text {. }
\end{aligned}
$$

Next, the derived subgroups and abelianisation of the Bieberbach groups with point group $C_{5}$ are presented in the following two lemmas.

Lemma 1. The derived subgroups of the Bieberbach groups with point group $C_{5}$ are given as :

$$
\begin{aligned}
& N_{1}(5)^{\prime}=\left\langle l_{1}^{-1} l_{4}^{-1}, l_{2}^{-2} l_{3}^{-1}, l_{1} l_{3}^{-1}, l_{1} l_{2}^{-1} l_{4}^{-1}\right\rangle \cong C_{0}{ }^{4}, \\
& N_{2}(6)^{\prime}=\left\langle l_{1}^{-1} l_{4}, l_{1} l_{2}^{-2} l_{3}^{-1}, l_{1}^{-1} l_{2} l_{3}^{-1}, l_{2}^{-1} l_{4}^{-1}\right\rangle \cong C_{0}^{4}, \\
& N_{3}(6)^{\prime}=\left\langle l_{2}^{-1} l_{4}, l_{3}^{-1} l_{5}^{-1}, l_{4}^{-1} l_{6}, l_{3} l_{6}^{-1}\right\rangle \cong C_{0}^{4} .
\end{aligned}
$$

\section{Proof.}

The Bieberbach group $N_{1}(5)$ is generated by 
elements $a, l_{1}, l_{2}, l_{3}, l_{4}$ and $l_{5}$ where $\left[a, l_{1}\right]=l_{1}^{-1} l_{4}^{-1}$, $\left[a, l_{2}\right]=l_{2}^{-2} l_{3}^{-1},\left[a, l_{3}\right]=l_{1} l_{3}^{-1},\left[a, l_{4}\right]=l_{1} l_{2}^{-1} l_{4}^{-1}$, $\left[a, l_{5}\right]=1$ and $\left[l_{i}, l_{j}\right]=1$ for all $1 \leq i<j \leq 5$. Then,

$$
N_{1}(5)^{\prime}=\left\langle l_{1}^{-1} l_{4}^{-1}, l_{2}^{-2} l_{3}^{-1}, l_{1} l_{3}^{-1}, l_{1} l_{2}^{-1} l_{4}^{-1}\right\rangle
$$

and is isomorphic to $C_{0}^{4}$ since $N_{1}(5)$ is torsionfree. Using similar method, the derived subgroups of $N_{2}(6)$ and $N_{3}(6)$ are found to be

$$
\begin{aligned}
& N_{2}(6)^{\prime}=\left\langle l_{1}^{-1} l_{4}, l_{1} l_{2}^{-2} l_{3}^{-1}, l_{1}^{-1} l_{2} l_{3}^{-1}, l_{2}^{-1} l_{4}^{-1}\right\rangle \cong C_{0}^{4}, \\
& N_{3}(6)^{\prime}=\left\langle l_{2}^{-1} l_{4}, l_{3}^{-1} l_{5}^{-1}, l_{4}^{-1} l_{6}, l_{3} l_{6}^{-1}\right\rangle \cong C_{0}^{4}
\end{aligned}
$$

Lemma 2. The abelianisation of the Bieberbach groups with point group $C_{5}$ are :

$$
\begin{aligned}
& N_{1}(5)^{a b}=\left\langle a N_{1}(5)^{\prime}, l_{1} N_{1}(5)^{\prime}\right\rangle \cong C_{0} \times C_{5}, \\
& N_{2}(6)^{a b}=\left\langle a N_{2}(6)^{\prime}, l_{1} N_{2}(6)^{\prime}, l_{6} N_{2}(6)^{\prime}\right\rangle \cong C_{0} \times C_{5} \times C_{0}, \\
& N_{3}(6)^{a b}=\left\langle a N_{3}(6)^{\prime}, l_{2} N_{3}(6)^{\prime}\right\rangle \cong C_{0}^{2} .
\end{aligned}
$$

\section{Proof.}

The abelianisation $\quad N_{1}(5)^{a b}$ is defined as $N_{1}(5)^{a b}=N_{1}(5) / N_{1}(5)^{\prime}$. Hence, it is generated by $a N_{1}(5)^{\prime}, l_{1} N_{1}(5)^{\prime}, l_{2} N_{1}(5)^{\prime}, l_{3} N_{1}(5)^{\prime}, l_{4} N_{1}(5)^{\prime} \quad$ and $l_{5} N_{1}(5)^{\prime}$. However, the generators are not independent. By the relations of $N_{1}(5), a^{5}=l_{5}$. Hence $a N_{1}(5)^{\prime} \cap l_{5} N_{1}(5)^{\prime}$ is not trivial, and $a N_{1}(5)^{\prime}=l_{5} N_{1}(5)^{\prime}$. Next, $\quad l_{1} N_{1}(5)^{\prime}=l_{3} N_{1}(5)^{\prime} \quad$ and $l_{4} N_{1}(5)^{\prime}=\left(l_{1} N_{1}(5)^{\prime}\right)^{-1} \quad$ since $\quad l_{1} l_{3}^{-1}, l_{1}^{-1} l_{4}^{-1} \in N_{1}(5)^{\prime}$. Besides, $\quad l_{1}\left(l_{2} l_{4}\right)^{-1}$ is also in $N_{1}(5)^{\prime}$. Thus, $l_{1} N_{1}(5)^{\prime}=\left(l_{2} l_{4}\right) N_{1}(5)^{\prime}$. By properties of a factor group $l_{1} N_{1}(5)^{\prime}=l_{2} N_{1}(5)^{\prime} l_{4} N_{1}(5)^{\prime}$. $\quad$ Then, since $l_{4} N_{1}(5)^{\prime}=\left(l_{1} N_{1}(5)^{\prime}\right)^{-1}, \quad$ it is shown that $l_{2} N_{1}(5)^{\prime}=\left(l_{1} N_{1}(5)^{\prime}\right)^{2}$. Therefore, the independent generators of $N_{1}(5)^{a b}$ are $a N_{1}(5)^{\prime}$ and $l_{1} N_{1}(5)^{\prime}$.

By the relations of $N_{1}(5)$, for any integer $r, a^{r}$ is not in $N_{1}(5)^{\prime}$. Since $N_{1}(5)^{\prime}$ is generated by elements of infinite order, then the order of $a N_{1}(5)^{\prime}$ is infinite. Meanwhile, it can be shown that $l_{1}^{5}=\left(l_{1} l_{3}^{-1}\right)\left(l_{1}^{-1} l_{4}^{-1}\right)^{-2}\left(l_{1} l_{2}^{-1} l_{4}^{-1}\right)^{2}\left(l_{2}^{-2} l_{3}^{-1}\right)^{-1}$ is in $N_{1}(5)^{\prime}$ since $l_{1} l_{3}^{-1}, l_{1}^{-1} l_{4}^{-1}, l_{1} l_{2}^{-1} l_{4}^{-1}, l_{2}^{-2} l_{3}^{-1} \in N_{1}(5)^{\prime}$.

This implies that $l_{1} N_{1}(5)^{\prime}$ has order 5. Therefore, $N_{1}(5)^{a b}=\left\langle a N_{1}(5)^{\prime}, l_{1} N_{1}(5)^{\prime}\right\rangle \cong C_{0} \times C_{5}$. Using similar method, the abelianisation of $N_{2}(6)$ and $N_{3}(6)$ are found to be

$N_{2}(6)^{a b}=\left\langle a N_{2}(6)^{\prime}, l_{1} N_{2}(6)^{\prime}, l_{6} N_{2}(6)^{\prime}\right\rangle \cong C_{0} \times C_{5} \times C_{0}$, $N_{3}(6)^{a b}=\left\langle a N_{3}(6)^{\prime}, l_{2} N_{3}(6)^{\prime}\right\rangle \cong C_{0}^{2}$.

Theorem 4. Let $N_{1}(5)$ be a Bieberbach group with point group $C_{5}$ of dimension five. Then, $\nabla\left(N_{1}(5)\right)=\left\langle a \otimes a, l_{1} \otimes l_{1},\left(a \otimes l_{1}\right)\left(l_{1} \otimes a\right)\right\rangle \cong C_{0} \times C_{5}^{2}$. Proof.

Based on Theorem 1, Proposition 3(i) and Lemma 2, $\nabla\left(N_{1}(5)\right) \quad$ is generated by $a \otimes a, l_{1} \otimes l_{1} \quad$ and $\left(a \otimes l_{1}\right)\left(l_{1} \otimes a\right)$. The abelianisation of $N_{1}(5)$ is denoted by $N_{1}(5)^{a b}$ with natural homomorphism $\epsilon: N_{1}(5) \rightarrow N_{1}(5)^{a b}$. Since $N_{1}(5)^{a b} \cong C_{0} \times C_{5}$, then by Proposition 7 ,

$$
\begin{aligned}
N_{1}(5)^{a b} \otimes & N_{1}(5)^{a b} \cong\left(C_{0} \times C_{5}\right) \otimes\left(C_{0} \times C_{5}\right) \\
& \cong\left(C_{0} \otimes\left(C_{0} \times C_{5}\right)\right) \times\left(C_{5} \otimes\left(C_{0} \times C_{5}\right)\right) \\
& \cong C_{0} \times C_{5} \times C_{5} \times C_{5} .
\end{aligned}
$$

Based on Lemma 2, $N_{1}(5)^{a b}$ is generated by $\epsilon(a)$ and $\epsilon\left(l_{1}\right)$ of order infinity and 5 , respectively. Again, by Proposition $\quad 7, \quad\langle\epsilon(a) \otimes \epsilon(a)\rangle \cong C_{0}$, $\left\langle\epsilon(a) \otimes \epsilon\left(l_{1}\right)\right\rangle \cong C_{5},\left\langle\epsilon\left(l_{1}\right) \otimes \epsilon(a)\right\rangle \cong C_{5}$, $\left\langle\epsilon\left(l_{1}\right) \otimes \epsilon\left(l_{1}\right)\right\rangle \cong C_{5}$. By Theorem 2, there is a natural epimorphism

$$
\alpha: N_{1}(5) \otimes N_{1}(5) \rightarrow N_{1}(5)^{a b} \otimes N_{1}(5)^{a b} .
$$

Therefore, the image $\alpha(a \otimes a)=\epsilon(a) \otimes \epsilon(a)$ has infinite order. Thus by Proposition 6, $a \otimes a$ has infinite order. 
Since $l_{1}^{5}$ is in $N_{1}(5)^{\prime}$, then $\left[l_{1}^{5},\left(l_{1}^{5}\right)^{\varphi}\right]=1$. Thus, by applying Proposition 4(i), $\left[l_{1}, l_{1}^{\varphi}\right]^{25}$ is trivial,

which implies that $\left[l_{1}, l_{1}^{\varphi}\right]$ has order dividing 25. Suppose the order of $\left[l_{1}, l_{1}^{\varphi}\right]$ is 25 , that is the smallest integer $m$ such that $\left[l_{1}, l_{1}^{\varphi}\right]^{m}=1$ is 25 . This is a contradiction since by Propositions 4 (i) and $5,\left[l_{1}, l_{1}^{\varphi}\right]^{10}=1$ due to the fact that $\left[l_{1}^{5}, l_{1}^{\varphi}\right]=\left[l_{1},\left(l_{1}^{5}\right)^{\varphi}\right]^{-1}$. Since the greatest common divisor of 25 and 10 is 5 , then the order of $\left[l_{1}, l_{1}^{\varphi}\right]$ is 5 . By Theorem $1, l_{1} \otimes l_{1}$ has order 5 .

Next, the order of $\left(a \otimes l_{1}\right)\left(l_{1} \otimes a\right)$ can be shown to be 5. By the identities of comutator, $\left(\left[a, l_{1}^{\varphi}\right]\left[l_{1}, a^{\varphi}\right]\right)^{5}=\left[a,\left(l_{1}^{5}\right)^{\varphi}\right]\left[l_{1}^{5}, a^{\varphi}\right] . \quad$ Again, since $l_{1}^{5} \in N_{1}(5)^{\prime}, \quad$ then $\left[l_{1}^{5}, a^{\varphi}\right]=\left[a,\left(l_{1}^{5}\right)^{\varphi}\right]^{-1}$. Hence, $\left(\left[a, l_{1}^{\varphi}\right]\left[l_{1}, a^{\varphi}\right]\right)^{5}=1$. Then, by Theorem 1 , the order of $\left(a \otimes l_{1}\right)\left(l_{1} \otimes a\right)$ is 5 . Therefore,

$$
\begin{aligned}
\nabla\left(N_{1}(5)\right) & =\left\langle a \otimes a, l_{1} \otimes l_{1},\left(a \otimes l_{1}\right)\left(l_{1} \otimes a\right)\right\rangle \\
& \cong C_{0} \times C_{5}^{2} .
\end{aligned}
$$

Similar method is applied to the other Bieberbach groups with point group $C_{5}$ and the results for all $\nabla\left(N_{i}(j)\right)$ are given in the following table.

Table 2: The homological functor $\nabla\left(N_{i}(j)\right)$

\begin{tabular}{|c|c|c|}
\hline$N_{i}(j)$ & $\nabla\left(N_{i}(j)\right)$ & $\cong$ \\
\hline$N_{1}(5)$ & $\left\langle a \otimes a, l_{1} \otimes l_{1}\right.$, & $C_{0} \times C_{3}$ \\
& $\left.\left(a \otimes l_{1}\right)\left(l_{1} \otimes a\right)\right\rangle$ & \\
\hline$N_{2}(6)$ & $\left\langle a \otimes a, l_{1} \otimes l_{1}, l_{6} \otimes l_{6}\right.$, & $C_{0}^{3} \times C_{5}^{3}$ \\
& $\left(a \otimes l_{1}\right)\left(l_{1} \otimes a\right)$, & \\
& $\left(a \otimes l_{6}\right)\left(l_{6} \otimes a\right)$, & \\
& $\left.\left(l_{1} \otimes l_{6}\right)\left(l_{6} \otimes l_{1}\right)\right\rangle$ & \\
\hline$N_{3}(6)$ & $\left\langle a \otimes a, l_{2} \otimes l_{2}\right.$, & $C_{0}^{3}$ \\
& $\left.\left(a \otimes l_{2}\right)\left(l_{2} \otimes a\right)\right\rangle$ & \\
\hline
\end{tabular}

\section{SUMMARY}

In this paper, the computation of homological invariant $\nabla(G)$ for all non-isomorphic Bieberbach groups with cyclic point groups of order three and five, up to dimension six, are presented. The results are summarized in Table 1 and Table 2. Since $\nabla(G)$ is a central subgroup of the nonabelian tensor square, then all the results are abelian and isomorphic to product of several copies of cyclic groups. 


\section{REFERENCES}

Abdul Ladi, N. F., Masri, R., Mohd Idrus, N., Sarmin, N. H. and Tan, Y. T. (2017) The central subgroups of the nonabelian tensor squares of some Bieberbach groups with elementary abelian 2-group point group. Jurnal Teknologi, 79(7), 115-121.

Blyth, R .D. and Fumagalli, F. and Morigi, M. (2010) Some structural results on the non-abelian tensor square of groups. Journal of Group Theory, 13, 83-94.

Blyth, R. D. and Morse, R .F. (2009) Computing the nonabelian tensor square of polycyclic groups. Journal of Algebra, 321, 2139-2148.

Brown, R., Johnson, D. L. and Robertson, E. F. (1987) Some computations of non-abelian tensor products of groups. J. of Algebra, 111, 177-202.

Ellis, E. and Leonard, F.(1995). Computing Schur multipliers and tensor products of finite groups. Proc. Roy. Irish Acad. Sect. A., 95(2), 137-147.

Masri, R. (2009) The nonabelian tensor squares of certain Bieberbach groups with cyclic point group of order two. PhD Thesis Department of Mathematics, Universiti Teknologi Malaysia.
Mat Hassim, H. I., Sarmin, N. H., Mohd Ali, Mohammad, S. A., Sarmin, N. H. and Mat Hassim, H. I. (2016) The Central Subgroup of the Nonabelian Tensor Square of a Torsion Free Space Group. AIP Conference Proceedings, 1750, 1-7.

Mohd Idrus, N., Wan Mohd Fauzi,W. N. F., Masri,R., Tan,Y. T., Sarmin, N. H. and Mat Hassim, H. I. (2015) The Central Subgroup of Nonabelian Tensor Square of the Third Bieberbach Group with Dihedral Point Group. International Journal of Applied Mathematics and Statistics, 53(4).

N. M. and Mohammad, S. A. (2014) The abelianisation of Bieberbach groups with cyclic point group of order three. Proceedings of $2^{\text {nd }}$ International Science Postgraduate Conference 2014., 907-919.

The CARAT homepage. (http://wwwb.math. rwthaachen.de/carat/).

Zomorodian, A. J. (2005) Topology for Computinge. Cambridge University Press. 